\title{
Accurate propagation of ultrashort pulses in nonlinear waveguides using propagation models for the analytic signal
}

O. Melchert, U. Morgner, B. Roth, I. Babushkin, A. Demircan

O. Melchert, U. Morgner, B. Roth, I. Babushkin, A. Demircan, "Accurate propagation of ultrashort pulses in nonlinear waveguides using propagation models for the analytic signal," Proc. SPIE 10694, Computational Optics II, 106940M (28 May 2018); doi: 10.1117/12.2313255

SPIE. Event: SPIE Optical Systems Design, 2018, Frankfurt, Germany 


\title{
Accurate propagation of ultrashort pulses in nonlinear waveguides using propagation models for the analytic signal
}

\author{
O. Melchert ${ }^{a}$ and U. Morgner ${ }^{a, b}$ and B. Roth ${ }^{a}$ and I. Babushkin ${ }^{b}$ and A. Demircan ${ }^{a, b}$ \\ ${ }^{a}$ Hannover Centre for Optical Technologies (HOT), 30167 Hannover, Germany; \\ ${ }^{b}$ Institute of Quantum Optics, Leibniz Universität Hannover, 30167 Hannover, Germany
}

\begin{abstract}
We present a numerical approach for the accurate simulation of the complex propagation dynamics of ultrashort optical pulses in nonlinear waveguides, especially valid for few-cycle pulses. The propagation models are derived for the analytical signal, which includes the real optical field, exempt from the commonly adopted slowly varying envelope approximation. As technical basis for the representation of the medium dispersion we use rational Padé approximants instead of commonly employed high-order polynomial expansions. The implementation of the propagation equation is based on the Runge-Kutta in the interaction picture method. In addition, our modular approach easily allows to incorporate a Raman response and dispersion in the nonlinear term. As exemplary use-cases we illustrate our numerical approach for the simulation of a few-cycle pulse at various center frequencies for an exemplary photonic crystal fiber and demonstrate the collision of a soliton and two different dispersive waves mediated by their group-velocity event horizon.
\end{abstract}

Keywords: Unidirectional field propagation, analytic signal, solitons, barrier scattering

\section{INTRODUCTION}

In this contribution we illustrate a numerical approach for the accurate simulation of the complex propagation dynamics of ultrashort optical pulses in nonlinear waveguides, especially valid for few-cycle pulses. The underlying propagation models are derived in terms of the analytical signal (AS) for the real optical field. ${ }^{1,2}$ They are exempt from the commonly adopted slowly varying envelope approximation but feature as prominent limiting case the envelope-based generalized nonlinear Schrödinger equation (GNLSE) with all usual effects. ${ }^{3}$ In addition, it is easy to amend the discussed propagation model to also feature a delayed Raman response. ${ }^{4}$ Subsequently we will particularly focus on the simplified forward model for the analytic signal (sFMAS), i.e. an unidirectional propagation equation that holds dispersion also in the nonlinear term. Among other applications, the considered propagation models have been used to illustrate a two-pulse collision scheme for the generation of supercontinuum (SC) spectra based on pulse reshaping at an intensity induced refractive index barrier, ${ }^{4}$ and revealing a soliton implosion phenomenon only observable in terms of a few cycle description ${ }^{5}$ for which the AS approach is especially suited.

As discussed in some detail below, the technical basis for the representation of the material dispersion is given by rational Padé approximants ${ }^{6}$ instead of the commonly employed high-order polynomial expansions, and the implementation of the propagation equation is based on an integrating factor solver ${ }^{7}$ adapted to the sFMAS. Both design choices assist to ameliorate the stiffness of the linear part of the model equation and allow for adequate evolution of the field up to large propagation distances.

As exemplary use-cases we consider: (i) the propagation of a few-cycle pulse including the Kerr effect and a delayed Raman response at various center frequencies for the refractive index profile of an "endlessly single mode" ${ }^{8,9}$ (ESM) photonic crystal fiber (PCF), and, (ii) the subsequent encounter of a soliton and two weak dispersive waves, i.e. demonstrating a scattering process involving three different center frequencies. In the latter setup, the collision process is mediated by a group velocity event horizon that forms through the nonlinear Kerr effect that accompanies the propagating light pulses and allows them to engage in an interaction. ${ }^{10-12}$ In such

Further author information: (Send correspondence to O.M.)

O.M.: E-mail: oliver.melchert@hot.uni-hannover.de

Computational Optics II, edited by Daniel G. Smith, Frank Wyrowski, Andreas Erdmann, Proc. of SPIE Vol. 10694, 106940M · C 2018 SPIE · CCC code: 0277-786X/18/\$18 · doi: 10.1117/12.2313255 
a setting, the nonlinear reflection of a comparatively weak probe pulse on a soliton pulse triggered a sequence of studies that aimed at providing a means of light control based on the concept of the optical group velocity horizon. ${ }^{4,13-16}$

The article is organized as follows: section 2 illustrates the propagation model and specifies the sFMAS, section 3 discusses the construction of the discrete AS, and section 4 summarizes the use-cases utilizing the ESM PCF. Finally, section 5 concludes with a summary.

\section{THE SIMPLIFIED FORWARD MODEL FOR THE ANALYTIC SIGNAL}

More than half a century ago the analytic signal was formally introduced by Gábor as a mathematical tool to analyze the information content of signals with application to communication engineering and, in particular, acoustics. ${ }^{17}$ While Gábor noted "... in physical acoustics a new formal approach to old problems cannot be expected to reveal much that is not already known ...", ${ }^{18}$ the introduction of the AS to time-frequency signal analysis, ${ }^{19}$ and, as discussed here, to the field of computational photonics bears great potential. Subsequently we illustrate a set of models for ultrashort pulse propagation via first-order propagation equations derived in terms of the $\operatorname{AS} \mathcal{E}(z, t)$ for the real optical field $E(z, t)$. In this regard it is most instructive to approach the problem in the spectral domain where the Fourier-transform $\hat{\mathcal{E}}(z, \omega)=\mathcal{F}[\mathcal{E}](z, \omega)$ of the AS is related to the Fourier-transform $\hat{E}(z, \omega)=\mathcal{F}[E](z, \omega)$ of the real optical field through ${ }^{1,15}$

$$
\hat{\mathcal{E}}(z, \omega)=[1+\operatorname{sgn}(\omega)] \hat{E}(z, \omega) .
$$

This readily demonstrates the characteristic property of the AS: it has a one-sided spectral definition, implying $\hat{\mathcal{E}}=0$ for $\omega<0$. For a detailed discussion of the subtleties involved in obtaining the AS in the time domain or the discrete case, where the latter is important when it comes to devising a numerical algorithm, see section 3 below. For the scalar second order nonlinear wave equation for the real electric field the AS naturally facilitates a separation of forward and backward propagating waves in the spectral domain, allowing to derive a set of first order nonlinear propagation equations that all match the pattern ${ }^{1}$

$$
i \partial_{z} \hat{\mathcal{E}}=-c_{L}(\omega) \hat{\mathcal{E}}-c_{N}(\omega) N(\mathcal{E}) .
$$

By adjusting the nonlinear function $N(\mathcal{E})$ as well as the coefficient functions $c_{L}(\omega)$ and $c_{N}(\omega)$ for the linear and nonlinear parts, Eq. (2) allows to simulate pulse propagation at a desired level of complexity. Subsequently we consider the simplified forward model for the analytic signal including the Raman effect (sFMAS-RA), specified by letting ${ }^{15}$

$$
\left.\begin{array}{l}
c_{\mathrm{L}}(\omega)=\left[\beta(\omega)-\omega / v_{0}\right], \\
c_{\mathrm{N}}(\omega)=n_{2} \omega / c_{0}, \\
N(\mathcal{E})=\mathcal{F}\left[\left(1-f_{R}\right)|\mathcal{E}|^{2} \mathcal{E}+f_{R} \mathcal{E} \hat{h}|\mathcal{E}|^{2}\right]_{\omega>0} .
\end{array}\right\} \quad \text { sFMAS-RA }
$$

Therein, $c_{L}(\omega)$ signifies a modified propagation profile at reference velocity $v_{0}, c_{0}$ is the speed of light, and $n_{2}$ refers to the index of nonlinear refraction. The propagation profile is related to the medium refractive index $n(\omega)$ via $\beta(\omega)=\omega \operatorname{Re}[n(\omega)] / c_{0}$. Further, $f_{R}$ denotes the fractional contribution of delayed Raman response, and $\hat{h}$ signifies convolution with a suitable Raman response function. Here, for our numerical experiments we consider an elaborate response function implementing the intermediate broadening model. ${ }^{20}$ For other choices and the level of complexity they entail, see appendix A. From a point of view of scientific software development, there are additional design choices that must be made in order to devise a numerical procedure for the above model system. These address the representation of the medium dispersion by a proper data structure and the propagation routine used to advance the AS.

\subsection{Modeling the medium refractive index}

For our numerical experiments we opt for an implementation of the medium refractive index via a Padéapproximant of order $m=4$ and $n=4$, given by

$$
n(\omega)=\frac{\sum_{n=0}^{4} p_{n} \omega^{n}}{1+\sum_{m=1}^{4} q_{m} \omega^{m}},
$$


(a)

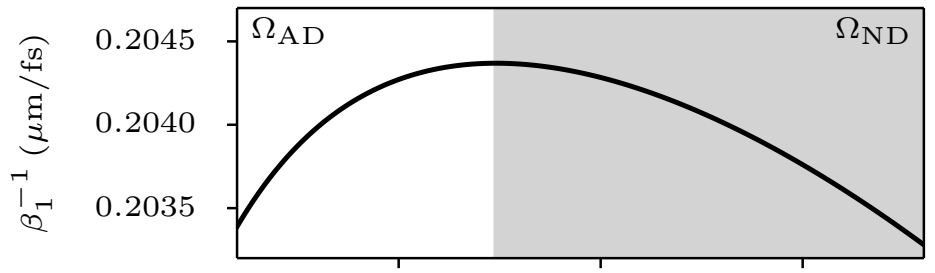

(b)

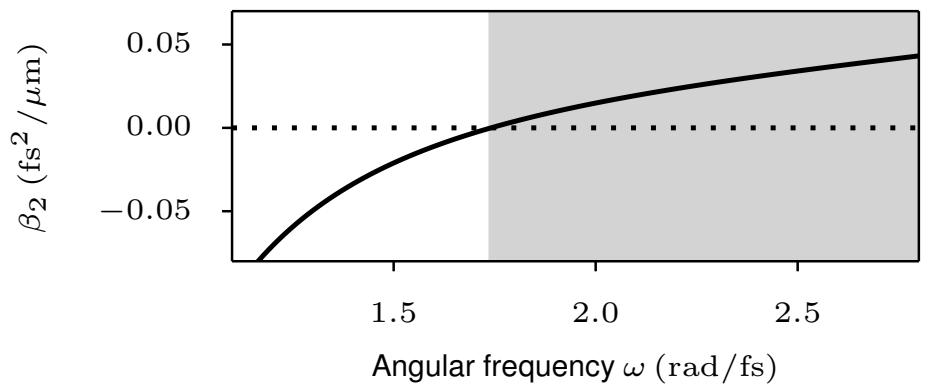

Figure 1. Illustration of (a) the group velocity $v_{\mathrm{G}}(\omega) \equiv \beta_{1}^{-1}(\omega)$, and (b) the group velocity dispersion $\beta_{2}(\omega)$ of the ESM PCF used for our numerical experiments. In both subplots the shaded area indicates the region of normal dispersion $\Omega_{\mathrm{ND}}$ (white background color indicates the region of anomalous dispersion $\Omega_{\mathrm{AD}}$ ).

with numerator and denominator coefficient sequences $p=(16.9,0.0,-319.1,0.0,34.8,0.0,-0.99,0.0,0.001)$ and $q=(1.0,0.0,-702.7,0.0,78.3,0.0,-2.34,0.0,0.006)$, respectively, wherein the coefficients of order $n$ have dimension $\mathrm{fs}^{n}$. The above refractive index models the $\mathrm{ESM}^{9}$ nonlinear photonic crystal fiber (PCF) and features a zero dispersion frequency at $\omega_{\text {ZDW }} \approx 1.741 \mathrm{rad} / \mathrm{fs}$, separating the regimes of anomalous dispersion (for $\omega<\omega_{\text {ZDW }}$; referred to as $\Omega_{\mathrm{AD}}$ ) and normal dispersion (for $\omega>\omega_{\mathrm{ZDW}}$; referred to as $\Omega_{\mathrm{ND}}$ ). Such a rational approximation of the medium refractive index allows to consider multiple pulses at different well separated center frequencies in a straight forward manner, see, e.g., section 4.2 below, and results in a more adequate approximation over a broad range of frequencies. ${ }^{6}$ It thus assists in overcoming the limitations of usual envelope-models that rely on a simple polynomial expansion of $n(\omega)$ in the vicinity of a chosen reference frequency ${ }^{21}$ and comprises a crucial ingredient to general propagation equations.

\subsection{Evolving the analytic signal}

For the propagation of an initial AS $\mathcal{E}_{0}(t) \equiv \mathcal{E}(t, z=0)$ we employ an integrating factor method ${ }^{7}$ that extends the idea underlying the popular GNLSE native "Runge-Kutta in the interaction picture method" 22 to the propagation equation Eq. (2). The implementation of the above numerical scheme follows a pseudospectral approach, wherein only the evaluation of the nonlinear function $N(\mathcal{E})$ is performed in the time domain. We further employ dealiasing to prevent numerical artifacts. Specific initial conditions studied for the ESM profile are detailed in section 4 below. During our numerical experiments we monitored conservation laws given by the period averaged energy and photon flux

$$
\begin{aligned}
& \mathrm{E}(z)=\sum_{\omega>0} c_{0} n(\omega) \frac{\epsilon_{0}|\hat{\mathcal{E}}(z, \omega)|^{2}}{2}, \quad \text { and } \\
& \mathrm{N}(z)=\sum_{\omega>0} \frac{c_{0} n(\omega)}{\omega} \frac{\epsilon_{0}|\hat{\mathcal{E}}(z, \omega)|^{2}}{2}
\end{aligned}
$$

respectively.

\section{GENERATION OF THE DISCRETE-TIME ANALYTIC SIGNAL}

As pointed out above, the AS signifies an advantageous tool that allows for a consistent description of ultrashort pulse propagation. While the application of the AS in a continuous setting is rather intuitive, its proper 
application to a discretized setting in terms of a numerical method requires some care. To illustrate this, we start by reviewing the key properties of the AS in the continuous case and subsequently allude to the problem of obtaining a discrete AS.

\subsection{Properties of the continuous analytic signal}

Consider a real valued electric field $E(t), t \in \mathbb{R}$, and its continuous-time Fourier-transform $\hat{E}(\omega), \omega \in \mathbb{R}$, both related by the transform pair $E(t)=\mathcal{F}^{-1}[\hat{E}](t)$ and $\hat{E}(\omega)=\mathcal{F}[E](\omega)$, wherein $\mathcal{F}[\cdot]$ and $\mathcal{F}^{-1}[\cdot]$ denote the forward and inverse Fourier transforms, respectively. If $E(t)$ exhibits special properties, $\hat{E}(\omega)$ reflects this. E.g., since $E(t)$ is unbounded with respect to $t, \hat{E}(\omega)$ is continuous. Further, since $E(t)$ is real valued, $\hat{E}(\omega)$ is complex conjugate symmetric, i.e. $\hat{E}(-\omega)=\hat{E}^{*}(\omega)$. In the time-domain, the continuous-time analytic signal $\mathcal{E}(t)$ corresponding to $E(t)$ is defined by the composition ${ }^{19}$

$$
\mathcal{E}(t)=E(t)+i \mathcal{H}[E](t),
$$

wherein $\mathcal{H}[E](t)$ signifies the Hilbert transform

$$
\mathcal{H}[E](t)=-\frac{1}{\pi} \lim _{\epsilon \rightarrow 0} \int_{\epsilon}^{\infty} \frac{E(t+\tau)-E(t-\tau)}{\tau} d \tau .
$$

Being of complex type, the AS might also be expressed as $\mathcal{E}(t)=|\mathcal{E}(t)| \exp \{i \phi(t)\}$, wherein $|\mathcal{E}(t)|$ and $\phi(t)$ denote the envelope and the instantaneous phase of the AS, respectively. The significance of the Hilbert transform must not be underestimated: it serves the generation of the AS and its action under Fourier transform ${ }^{23}$

$$
\mathcal{F}[\mathcal{H}[E]](\omega)=-i \operatorname{sgn}(\omega) \mathcal{F}[E](\omega)
$$

allows the AS in the frequency domain to assume a much more appealing form. I.e., in effect the action of the Hilbert transform consists in multiplying the Fourier-transform of its argument by the multiplier $-i \operatorname{sgn}(\omega)$, corresponding to a phase shift of $-\pi / 2$ for positive frequencies and $\pi / 2$ for negative frequencies. Likewise, the term $i \mathcal{H}[E](t)$ leaves the positive frequency components unaltered and shifts the negative ones by $\pi$. Hence, it does not come as a surprise that the Fourier-transform $\hat{\mathcal{E}}(\omega)$ of the AS can be cast into the form of Eq. (1), demonstrating that the spectrum of the AS is related to the spectrum of the real electric field alone. In contrast, the construction of the time-domain AS requires an additional integration according to Eq. (7). ${ }^{17}$ In particular, Eq. (1) displays that in case of a continuous-time real signal one can give a simple two-step recipe to obtain the frequency domain representation of the AS: "Suppress the amplitudes belonging to negative frequencies, and multiply the amplitudes of positive frequencies by two." as Gábor summarized. ${ }^{17}$

A related problem, important from a point of view of devising reliable algorithms for the generation of the AS, is to test whether a function $f(t)$ indeed represents an AS. Therefore, Eqs. (7) and (1) imply two distinct properties of the AS that are ideally suited as unit-tests for appropriate software components: (i) if $f(t)$ represents the analytic signal corresponding to a real-valued function $g(t)$, then $g(t)=\operatorname{Re}[f(t)]$, and, (ii) due to the orthogonality between the real and imaginary parts of $f(t)$ imposed by the action of the Hilbert transform underlying the definition of $\operatorname{Im}[f(t)]$, we are led to expect that $\int_{-\infty}^{\infty} \operatorname{Re}[f(t)] \operatorname{Im}[f(t)] d t=0$.

\subsection{Obtaining a proper discrete analytic signal}

In the preceding subsection, a general recipe for obtaining a continuous-time frequency-domain AS was discussed. While this is fine in the realm of a continuous mathematical model, it fails in case of a discrete and bounded computational domain that accompanies any practical numerical solution procedure. ${ }^{24}$ As a consequence, in a discretized setting the amplitudes of the AS frequency components need to be altered slightly. To specify how, consider a discrete real signal $E(n)=E\left(t_{n}\right)$, sampled at, say, equidistant mesh points $t_{n}=t_{0}+n \Delta t$ with $n=0, \ldots, N-1$ on a $1 \mathrm{D}$ grid, and its discrete-time Fourier transform (DTFT) $\hat{E}(m)=\hat{E}\left(\omega_{m}\right)$, with $\omega_{m}=2 \pi m /(N \Delta t)$. Then, a proper discrete AS in the frequency domain is obtained by setting

$$
\hat{\mathcal{E}}(m)= \begin{cases}\hat{E}(m), & \text { for } m=0, N / 2 \\ 2 \hat{E}(m), & \text { for } 1 \leq m \leq N / 2-1 \\ 0, & \text { for } N / 2+1 \leq m \leq N-1\end{cases}
$$


(a)

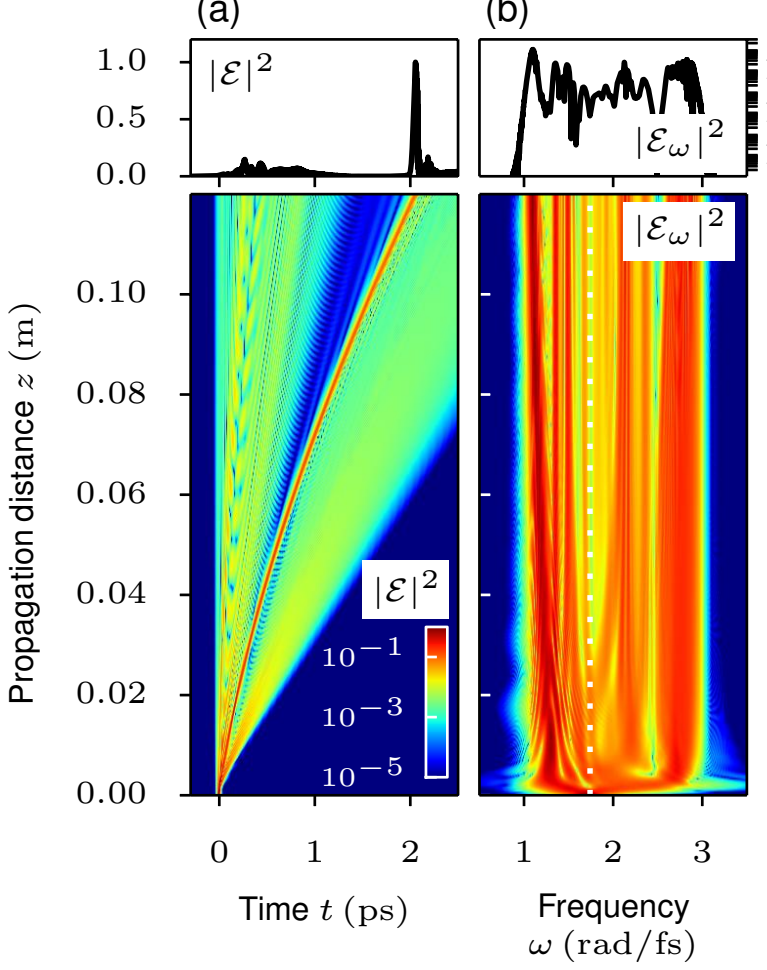

(c)

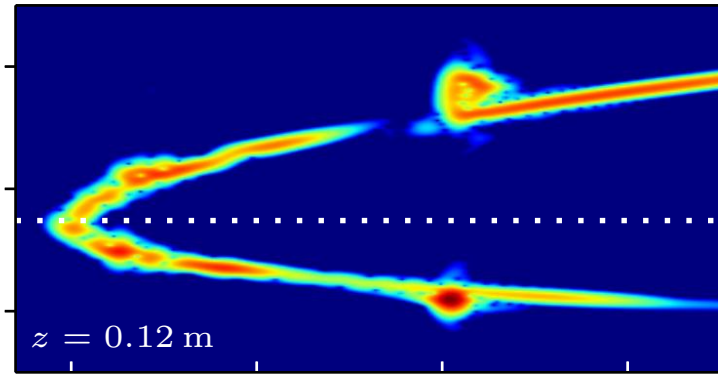

3

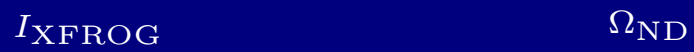

2

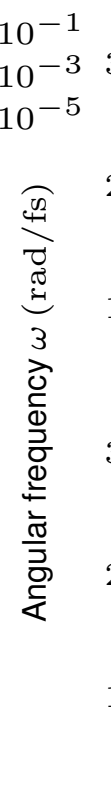

1

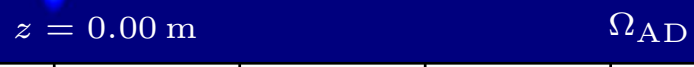

0

1

2

3

Delay $\tau(\mathrm{ps})$

Figure 2. Evolution of the AS for a $\tau=7 \mathrm{fs}$ soliton pulse of order $N=8$ at a central frequency $\omega=1.7 \mathrm{rad} / \mathrm{fs}$ in $\Omega_{\mathrm{AD}}$. (a) from top to bottom: normalized squared modulus of the AS at the propagation distance $z=0.12 \mathrm{~m}$ and evolution of the AS in the time domain. (b) from top to bottom: normalized squared modulus spectrum of the AS at $z=0.12 \mathrm{~m}$ and its evolution. (c) cross-correlation XFROG-traces of signals at selected propagation distances $z$.

and its time domain representation can be found from the inverse DTFT. As evident from Eq. (10), the frequencies at $m=0$ and $N / 2$ assume a distinguished role. This can be attributed to the requirement of the DTFT of $\operatorname{Im}[\mathcal{E}]$ to be a periodic and odd function. ${ }^{24}$ While it is straightforward to see that the above prescription suppresses negative frequency components, it is not immediately obvious that it yields a proper AS. As a remedy, the unit-tests pointed out earlier can be used to verify Eq. (10). They further spot the inadequacy of the continuous recipe Eq. (1) if erroneously applied to the discrete setting.

Note that Python's ${ }^{25}$ Scipy $^{26}$ library offers a mapping of the above discrete AS recipe to a software implementation, employing the above frequency-domain approach. In the Scipy ecosystem, the respective function is located in the submodule signal and is available as scipy.signal.hilbert*

\section{EXEMPLARY APPLICATIONS}

Subsequently, so as to illustrate an application of the propagation models based on evolving the AS, we present two use-cases that accentuate different challenges for any propagation approach. In subsection 4.1 we illustrate the propagation of a short, few-cycle pulse over an extended z-range, and, in subsection 4.2 we demonstrate the propagation of an initial condition that involves several pulses at different center frequencies.

\subsection{Propagation of an intense few-cycle pulse}

As first use-case we consider an initial condition consisting of a single soliton with duration $\tau=7 \mathrm{fs}(\approx 3.8$ cycles) and soliton order $N_{\mathrm{s}}=8$, prepared at the three different center frequencies $\omega=1.7,1.741$, and, 2.5 rad/fs

${ }^{*}$ Albeit the function is referred to as hilbert it allows to obtain the discrete analytic signal for a real-valued discrete input array. 
(a)

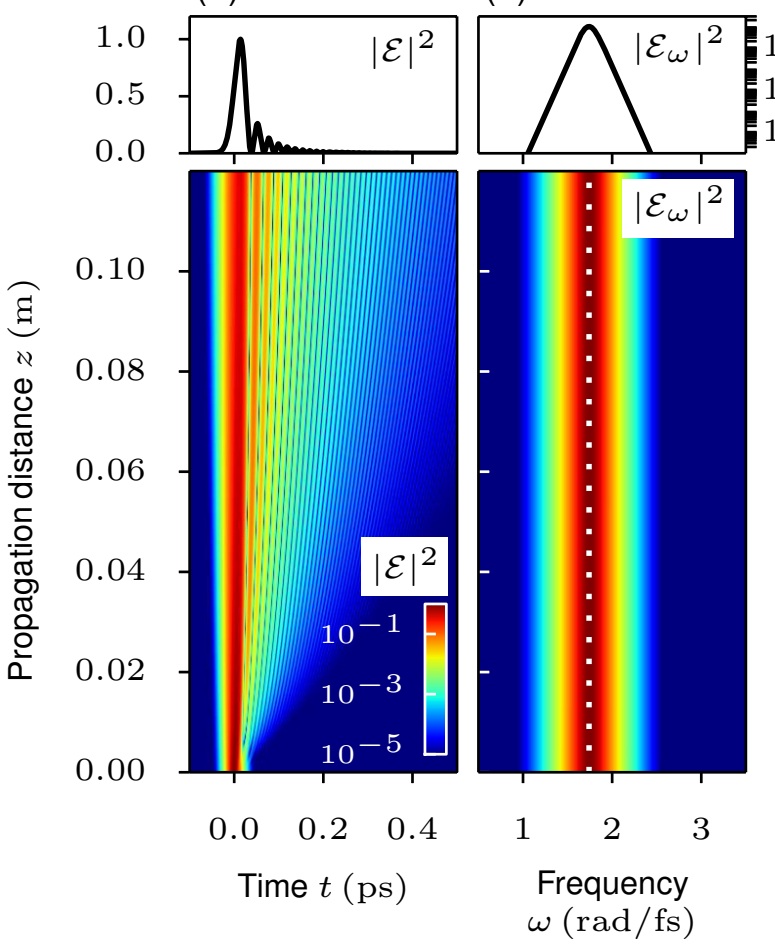

(c)

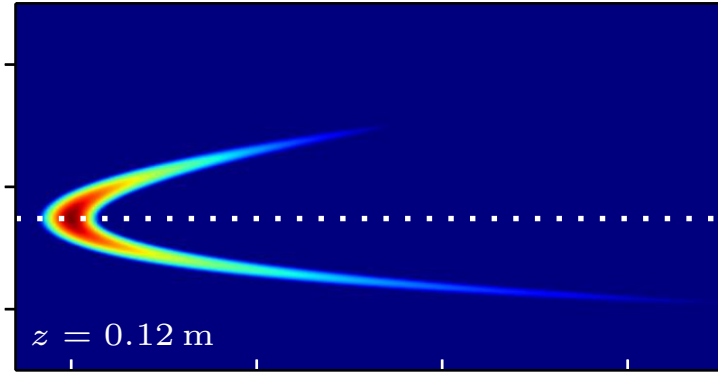

3

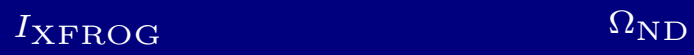

2

1

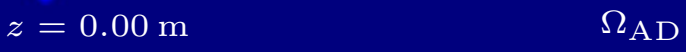

0
3

Figure 3. Evolution of the AS for a $\tau=7$ fs soliton pulse of order $N=8$ at the zero-dispersion frequency $\omega_{\mathrm{ZDW}} \approx$ $1.741 \mathrm{rad} / \mathrm{fs}$. (a) from top to bottom: normalized squared modulus of the AS at the propagation distance $z=0.12 \mathrm{~m}$ and evolution of the AS in the time domain. (b) from top to bottom: normalized squared modulus spectrum of the AS at $z=0.12 \mathrm{~m}$ and its evolution. (c) cross-correlation XFROG-traces of signals at selected propagation distances $z$.

with a dynamics governed by the propagation profile of the ESM PCF discussed above. The evolution of the corresponding initial condition is monitored in a co-moving frame of reference at speed $v_{0}=v_{\mathrm{G}}(\omega)$. As initial electric field we use

$$
E(z=0, t)=\operatorname{Re}\left[\Psi_{0} \operatorname{sech}(t / \tau) e^{-i \omega t}\right]
$$

with $\Psi_{0}$ determined by the condition $\Psi_{0}^{2}=N_{\mathrm{s}}^{2} c_{0} n_{2}\left|\beta_{2}(\omega)\right| /\left(\tau^{2} \omega\right)$. To facilitate a propagation using the sFMASRA, we obtain the AS for the above initial condition following Eq. (10). The computational domain is restricted to the interval $|t|<4$ ps using $N_{t}=2^{14}$ equidistant mesh points. In all simulations reported below we set $n_{2}=3 \cdot 10^{-8} \mu \mathrm{m}^{2} / \mathrm{W}$ and $f_{R}=0.18$, i.e. the values for fused silica fibers. ${ }^{21}$

The propagation of the AS for the above three parameter settings are summarized in Figs. 2-4. So as to assist in disentangling the time-frequency relationships of the propagating analytic signal, we complement its temporal and spectral evolution via cross-correlation XFROG traces, see appendix B. The evolution of the AS for $\omega=1.7 \mathrm{rad} / \mathrm{fs}$, i.e. in the region of anomalous dispersion, is illustrated in Fig. 2. As can be seen from the figure, the close proximity of the central frequency to the onset of the region of normal dispersion causes much of the available energy to convert to radiation. As a consequence, only a single soliton emerges from the initial condition upon propagation. This behavior is distinct from the usual soliton fission process, where, depending on the input soliton order $N_{\mathrm{s}}$, the initial pulse breaks up into several fundamental solitons that subsequently experience self-frequency shifts that are characteristic for their individual temporal duration and propagation in presence of Raman scattering. Also note the interaction of the continuously decelerating soliton with dispersive waves in the frequency range $\omega=2.5-3.2 \mathrm{rad} / \mathrm{fs}$ in $\Omega_{\mathrm{ND}} \cdot{ }^{27}$ The propagation dynamics right at the zero-dispersion frequency is shown in Fig. 3. Therein, the characteristics of the underlying refractive index profile in the vicinity 
(a)

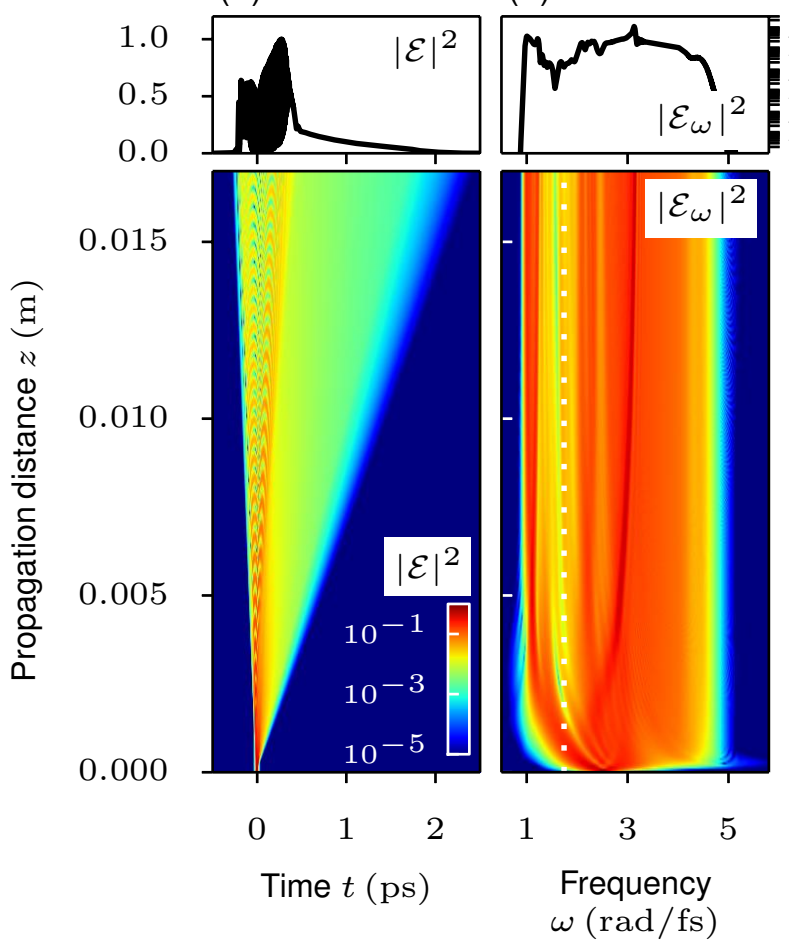

(c)

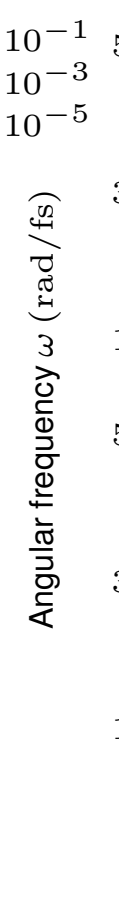

5

3

3
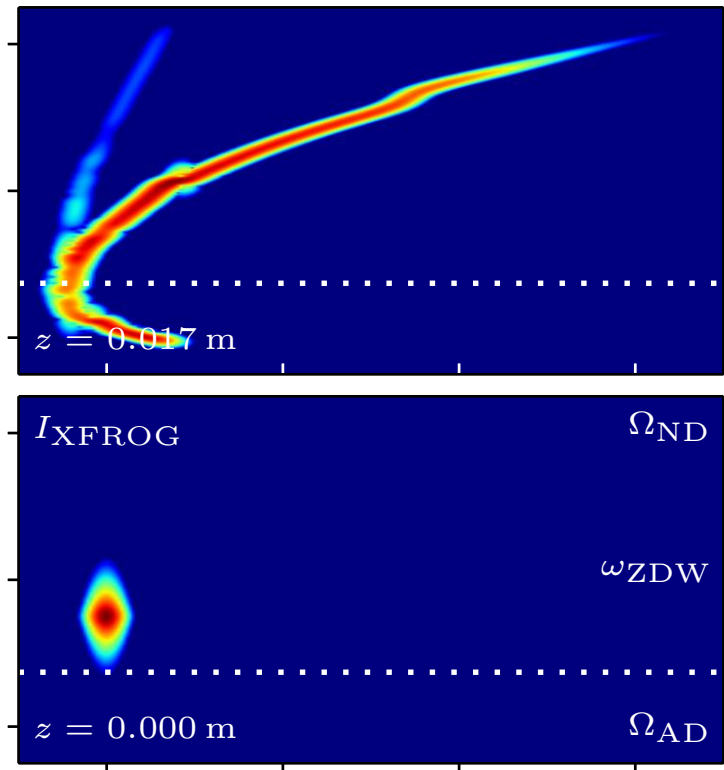

0

1

2

3

Delay $\tau(\mathrm{ps})$

Figure 4. Evolution of the AS for a $\tau=7$ fs soliton pulse of order $N=8$ at a central frequency $\omega=2.5 \mathrm{rad} / \mathrm{fs}$ deep in $\Omega_{\mathrm{ND}}$. (a) from top to bottom: normalized squared modulus of the AS at the propagation distance $z=0.017 \mathrm{~m}$ and evolution of the AS in the time domain. (b) from top to bottom: normalized squared modulus spectrum of the $\mathrm{AS}$ at $z=0.017 \mathrm{~m}$ and its evolution. (c) cross-correlation XFROG-traces of signals at selected propagation distances $z$.

of $\omega_{\text {ZDW }}$ and the large spectral width of the 7 fs pulse cause the strong distortion of the profile at positive time delays. Finally, Fig. 4 summarizes the evolution of the initial condition deep in the region of normal dispersion at $\omega=2.5 \mathrm{fs}$. As evident from the propagation of the squared magnitude of the AS in the time domain and the XFROG traces, a steep shock front in the leading part of the pulse is formed. However, note that due to the subtleties of the underlying refractive index profile and the distance of the center frequency to the onset of anomalous dispersion, no soliton is ignited.

\subsection{Collision of a soliton with two dispersive waves}

As second use-case we consider the collision of a soliton (S) and two dispersive waves (DW1 and DW2) at two different center-frequencies in the collinear setup DW2-DW1-S. Initially, S has center frequency $\omega_{\mathrm{S}}=1.5 \mathrm{rad} / \mathrm{fs}_{\mathrm{S}}$ and duration $\tau_{\mathrm{S}}=20 \mathrm{fs}$, and is initially at rest in a co-moving frame of reference at speed $v_{0}=v_{\mathrm{G}}\left(\omega_{\mathrm{S}}\right)=$ $0.20427 \mu \mathrm{m} / \mathrm{fs}$. The dispersive waves are characterized by $\omega_{\mathrm{DW} 1}=2.06 \mathrm{rad} / \mathrm{fs}$, injected with a time advance of $\Delta t_{1}=500 \mathrm{fs}$, and, $\omega_{\mathrm{DW} 2}=2.05 \mathrm{rad} / \mathrm{fs}$, injected with a time advance of $\Delta t_{1}=1000 \mathrm{fs}$. Both DWs have duration $\tau=60 \mathrm{fs}$ and amplitude ratio 0.3 relative to $\mathrm{S}$. As initial electric field we thus use

$$
E(z=0, t)=\operatorname{Re}\left[\Psi_{\mathrm{S}} \operatorname{sech}\left(\frac{t}{\tau_{\mathrm{S}}}\right) e^{-i \omega_{\mathrm{S}} t}+\Psi_{\mathrm{DW} 1} \operatorname{sech}\left(\frac{t+\Delta t_{1}}{\tau_{\mathrm{DW} 1}}\right) e^{-i \omega_{\mathrm{DW} 1} t}+\Psi_{\mathrm{DW} 2} \operatorname{sech}\left(\frac{t+\Delta t_{2}}{\tau_{\mathrm{DW} 2}}\right) e^{-i \omega_{\mathrm{DW} 2} t}\right],
$$

with $\Psi_{\mathrm{S}}$ and $\Psi_{\mathrm{DW} 1}=\Psi_{\mathrm{DW} 2}=0.3 \Psi_{\mathrm{S}}$ determined by the condition $\Psi_{\mathrm{S}}^{2}=c_{0} n_{2}\left|\beta_{2}\left(\omega_{\mathrm{S}}\right)\right| /\left(\tau_{\mathrm{S}}^{2} \omega_{\mathrm{S}}\right)$ for a fundamental soliton. Again, we obtain the initial AS by using Eq. (10). The computational domain is restricted to the interval 
(a)

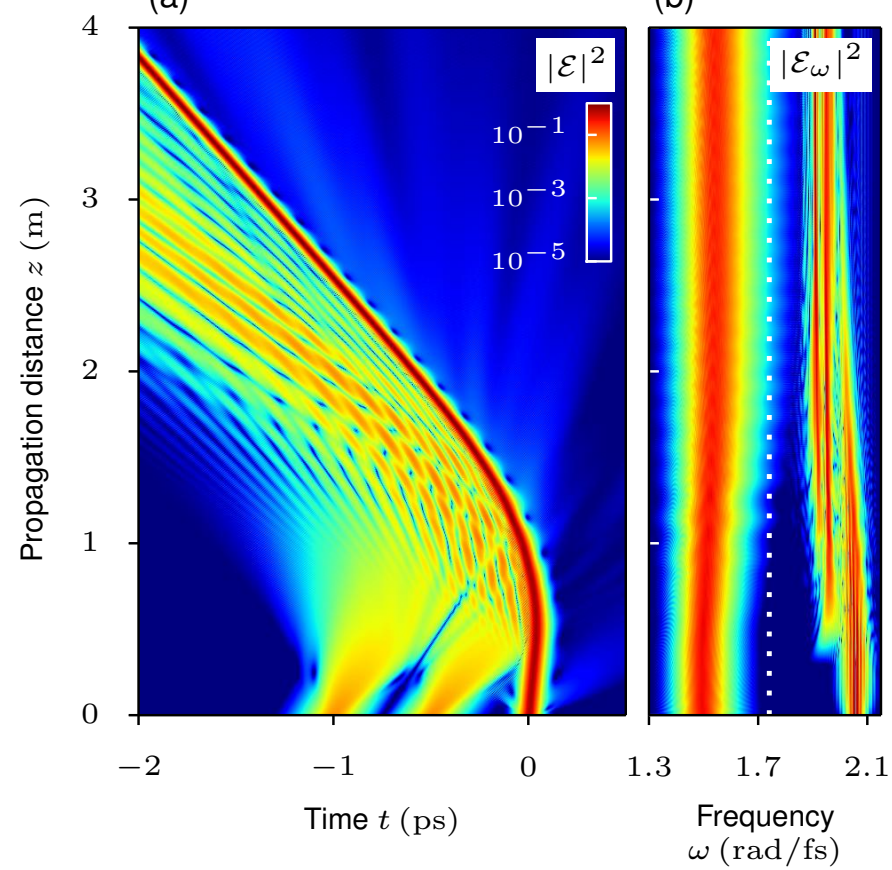

(c)

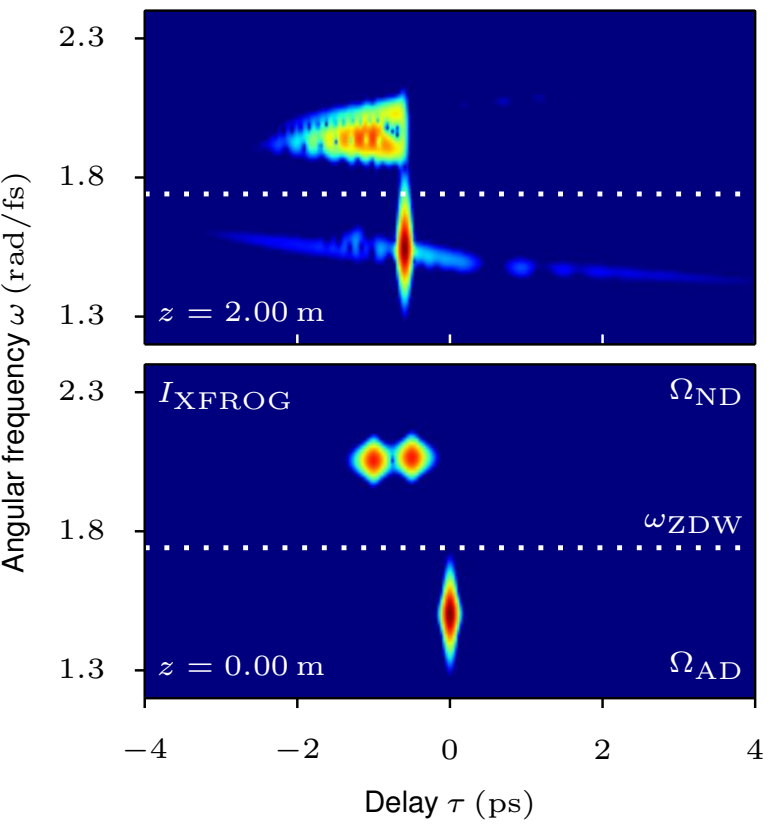

Figure 5. Scattering process of a soliton (S) and two dispersive waves (DWs) in the collinear setup DW2-DW1-S. The soliton initially propagates with a center frequency $\omega_{\mathrm{S}}=1.5 \mathrm{rad} / \mathrm{fs}$ and has an initial duration of $\tau_{\mathrm{S}}=20 \mathrm{fs}$. The dispersive waves are characterized by $\omega_{\mathrm{DW} 1}=2.06 \mathrm{rad} / \mathrm{fs}$, injected with a time advance of $\Delta t_{1}=500 \mathrm{fs}$, and, $\omega_{\mathrm{DW} 2}=2.05 \mathrm{rad} / \mathrm{fs}$, injected with a time advance of $\Delta t_{1}=1000 \mathrm{fs}$. Both DWs have duration $\tau=60 \mathrm{fs}$ and amplitude ratio 0.3 relative to S. (a) squared modulus of the analytic signal in the time domain, (b) squared modulus spectrum of the analytic signal, and, (c) cross-correlation XFROG-traces of signals at selected propagation distances $z$.

$|t|<8$ ps using $N_{t}=2^{15}$ equidistant mesh points. For our numerical experiment we used $n_{2}=3 \cdot 10^{-8} \mu \mathrm{m}^{2} / \mathrm{W}$ and neglected the Raman effect.

Note that under the propagation profile of the ESM, a dispersive wave is initially group velocity matched to $\mathrm{S}$ at $\omega^{\prime} \approx 2.019 \mathrm{rad} / \mathrm{fs}$, see Fig. 1(a). A dispersive wave prepared at a center frequency $\omega_{\mathrm{DW}}>\omega^{\prime}$ is initially slower than S. Hence, the ESM exhibits a convex group velocity profile, a central feature required for the creation of a group velocity event horizon supporting the above collision process. ${ }^{14}$ Also note that the underlying propagation equation does not rely on the introduction of a specific carrier frequency. Especially in a setting comprising several pulses at different center frequencies, introduction of such a carrier frequency represents a severe constraint restricting the applicability of naive propagation models.

The intricate dynamics of the above setup is summarized in Fig. 5. As evident from the time evolution of the initial condition shown in Fig. 5(a), S catches up with DW1 and engages in an interaction that results in a partial reflection of DW1 and an acceleration of both pulses. ${ }^{15}$ Depending on the group velocity mismatch between both, the center frequency of the reflected part of DW1 experiences a frequency down-conversion, see Fig. 5(b), accompanied by an increase of its group velocity. As a consequence, the center frequency of $\mathrm{S}$ necessarily increases to obey conservation of the photon flux, in the frequency domain expressed as $\mathrm{N}(z)=$ $\sum_{\omega>0} c_{0} \epsilon_{0}(2 \omega)^{-1} n(\omega)\left|\mathcal{E}_{\omega}(z)\right|^{2}=$ const. $^{1}$ In effect, the soliton reacts to the scattering event by adjusting its center frequency and temporal width to conform to a global energy and momentum conservation constraint. This above process is repeated upon the mutual encounter of S and DW2. In summary, the center frequency of $\mathrm{S}$ increases from its initial value $\omega_{\mathrm{S}}=1.5 \mathrm{rad} / \mathrm{fs}$ to $\omega_{\mathrm{S}}^{\prime}=1.55 \mathrm{rad} / \mathrm{fs}$ above the propagation distance $z=2 \mathrm{~m}$. This corresponds to an increase of its group velocity from $v_{0}=0.20427 \mu \mathrm{m} / \mathrm{fs}$ to $v_{\text {fin }}=0.20430 \mu \mathrm{m} / \mathrm{fs}$. Also, its width decreases from $\tau_{\mathrm{S}}=20 \mathrm{fs}$ to $\tau_{\mathrm{S}}^{\prime}=14.2 \mathrm{fs}$. Finally, note the appearance of small oscillations in the soliton 
width in both, time and frequency domain. These are a result of the spectral recoil experienced by $\mathrm{S}$ whenever its center frequency comes too close to $\omega_{\mathrm{ZDW}}{ }^{28}$

\section{SUMMARY}

We illustrated a numerical approach for the accurate simulation of the complex propagation dynamics of ultrashort optical pulses based on the analytic signal for the real optical field. The underlying propagation models are exempt from the commonly adopted slowly varying envelope approximation and are also valid in cases where the considered field pulses might consist of few cycles only.

As exemplary use-cases we considered the propagation of a short $7 \mathrm{fs}$ pulse in terms of the simplified forward model for the analytic signal including the Raman effect and illustrated the propagation characteristics of the AS for different choices of the pulse center frequency for the propagation profile of an ESM PCF. In addition we revisited a scattering process involving a soliton and multiple dispersive waves ${ }^{29}$ in which the intricate collision dynamics is mediated by a group velocity event horizon that forms through the nonlinear Kerr effect that accompanies the propagating light pulses.

\section{APPENDIX A. PROPAGATION MODELS FOR THE ANALYTIC SIGNAL}

Adjusting the nonlinear function $N(\mathcal{E})$ as well as the coefficient functions $c_{L}(\omega)$ and $c_{N}(\omega)$ for the linear and nonlinear part of Eq. (2) allows to specify different propagation models for the AS. E.g. the forward model for the AS (FMAS), featuring dispersion in the nonlinear part, might be obtained by letting 6

$$
\left.\begin{array}{l}
c_{\mathrm{L}}(\omega)=\left[\beta(\omega)-\omega / v_{0}\right], \\
c_{\mathrm{N}}(\omega)=3 \omega^{2} \chi /\left(8 c_{0}^{2} \beta(\omega)\right), \\
N(\mathcal{E})=\mathcal{F}\left[|\mathcal{E}|^{2} \mathcal{E}\right]_{\omega>0} .
\end{array}\right\} \quad \text { FMAS }
$$

with $c_{L}(\omega)$ signifying a modified propagation profile at the reference velocity $v_{0}, c_{0}$ the speed of light, and $\chi$ indicating the nonlinear susceptibility. A simplified variant of the forward model for the AS, termed sFMAS, might be obtained by setting ${ }^{15}$

$$
\left.\begin{array}{l}
c_{\mathrm{L}}(\omega)=\left[\beta(\omega)-\omega / v_{0}\right], \\
c_{\mathrm{N}}(\omega)=n_{2} \omega / c_{0}, \\
N(\mathcal{E})=\mathcal{F}\left[|\mathcal{E}|^{2} \mathcal{E}\right]_{\omega>0},
\end{array}\right\} \quad \text { sFMAS }
$$

wherein $n_{2}$ refers to the index of nonlinear refraction. Amending $N(\mathcal{E})$ in the definition of the sFMAS above by a suitable delayed Raman response function yields the propagation model detailed in section 2 .

\section{APPENDIX B. CALCULATION OF XFROG TRACES}

As a numerical tool allowing to disentangle the time-frequency composition of the analytic signal upon propagation, we employ an XFROG analysis. ${ }^{30}$ Therefore, a spectrally resolved crosscorrelation of the analytic signal $\mathcal{E}(z, t)$ is computed employing a short time Fourier transform via a Gaussian window function $E_{\text {ref }}(t)$, centered at $t$ and having root mean square width $\sigma$. The respective squared magnitude

$$
I_{\mathrm{XFROG}}(z, \omega, \tau)=\left|\int_{-\infty}^{\infty} \mathcal{E}(z, t) E_{\mathrm{ref}}(t-\tau) \exp \{i \omega t\} \mathrm{d} t\right|^{2},
$$

wherein $\tau$ refers to the delay of the reference signal $E_{\text {ref }}$ relative to the test pulse $\mathcal{E}$, yields the XFROG trace at a given propagation distance $z$. In any such analysis, the width $\sigma$ of the reference pulse needs to be adjusted so as to yield a reasonable tradeoff between delay-time and frequency resolution. Here, we used the fixed value of $\sigma=50 \mathrm{fs}$ in all our analyses. 


\section{ACKNOWLEDGMENTS}

This research work received funding from the VolkswagenStiftung within the Niedersachsisches Vorab program in the framework of the project Hybrid Numerical Optics (HYMNOS; Grant ZN 3061).

\section{REFERENCES}

[1] Amiranashvili, S. and Demircan, A., "Hamiltonian structure of propagation equations for ultrashort optical pulses," Phys. Rev. A 82, 013812 (2010).

[2] Amiranashvili, S. and Demircan, A., "Ultrashort Optical Pulse Propagation in terms of Analytic Signal," Advances in Optical Technologies 2011, 989515 (2011).

[3] Agrawal, G. P., [Nonlinear Fiber Optics], Academic Press, Boston (2013).

[4] Demircan, A., Amiranashvili, S., Brée, C., Mahnke, C., Mitschke, F., and Steinmeyer, G., "Rogue events in the group velocity horizon," Scientific Reports 2, 850 (2012).

[5] Babushkin, I., Tajalli, A., Sayinc, H., Morgner, U., Steinmeyer, G., and Demircan, A., "Simple route toward efficient frequency conversion for generation of fully coherent supercontinua in the mid-IR and UV range," Light: Science 83 Applications 6, e16218 (2017).

[6] Amiranashvili, S., Bandelow, U., and Mielke, A., "Padé approximant for refractive index and nonlocal envelope equations," Opt. Commun. 283, 480 - 485 (2010).

[7] Milewski, P. A. and Tabak, E. G., "A PseudoSpectral Procedure for the Solution of Nonlinear Wave Equations with Examples from Free-Surface Flows," SIAM Journal on Scientific Computing 21, 1102-1114 (1999).

[8] Birks, T. A., Knight, J. C., and Russell, P. S. J., "Endlessly single-mode photonic crystal fiber," Opt. Lett. 13, 961-963 (1997).

[9] Stone, J. M. and Knight, J. C., "Visibly 'white' light generation in uniform photonic crystal fiber using a microchip laser," Optics Express 16, 2670 (2007).

[10] Wang, S. F., Mussot, A., Conforti, M., Bendahmane, A., Zheng, X. L., and Kudlinski, A., "Optical event horizons from the collision of a soliton and its own dispersive wave," Phys. Rev. A 92, 023837 (2015).

[11] Philbin, T. G., Kuklewicz, C., Robertson, S., Hill, S., König, F., and Leonhardt, U., "Fiber-Optical Analog of the Event Horizon," Science 319, 1367-1370 (2008).

[12] Stegeman, G. I. and Segev, M., "Optical Spatial Solitons and Their Interactions: Universality and Diversity," Science 286(5444), 1518-1523 (1999).

[13] Hill, S., Kuklewicz, C. E., Leonhardt, U., and König, F., "Evolution of light trapped by a soliton in a microstructured fiber," Opt. Express 17, 13588-13601 (2009).

[14] Demircan, A., Amiranashvili, S., and Steinmeyer, G., "Controlling Light by Light with an Optical Event Horizon," Phys. Rev. Lett. 106, 163901 (2011).

[15] Demircan, A., Amiranashvili, S., Brée, C., Morgner, U., and Steinmeyer, G., "Supercontinuum generation by multiple scatterings at a group velocity horizon," Opt. Express 22, 3866-3879 (2014).

[16] Choudhary, A. and König, F., "Efficient frequency shifting of dispersive waves at solitons," Optics Express 20, 5538-5546 (2012).

[17] Gabor, D., "Theory of communication," J. Inst. Electr. Eng. 93, 429 - 441 (1946).

[18] Gabor, D., "Acoustical Quanta and the Theory of Hearing," Nature 159, 591 - 594 (1947).

[19] Boashash, B. and Barkat, B., [Wavelet Transforms and Time-Frequency Signal Analysis. Applied and Numerical Harmonic Analysis], ch. Introduction to Time-Frequency Signal Analysis, 321-380. In Debnath ${ }^{23}$ (2001).

[20] Hollenbeck, D. and Cantrell, C. D., "Multiple-vibrational-mode model for fiber-optic Raman gain spectrum and response function," J. Opt. Soc. Am. B 19, 2886 (2002).

[21] Dudley, J. M., Genty, G., and Coen, S., "Supercontinuum generation in photonic crystal fiber," Rev. Mod. Phys. 78, 1135-1184 (2006).

[22] Hult, J., "A Fourth-Order Runge-Kutta in the Interaction Picture Method for Simulating Supercontinuum Generation in Optical Fibers," J. Lightwave Technol. 25, 3770-3775 (2007). 
[23] Debnath, L., ed., [Wavelet Transforms and Time-Frequency Signal Analysis. Applied and Numerical Harmonic Analysis], Birkhäuser Boston, Boston, MA (2001).

[24] Marple, S. L., "Computing the discrete-time "analytic" signal via FFT," IEEE Trans. Signal Processing 47, 2600-2603 (1999).

[25] Rossum, G., "Python Reference Manual," tech. rep., Amsterdam, The Netherlands, The Netherlands (1995).

[26] Jones, E., Oliphant, T., Peterson, P., et al., "SciPy: Open source scientific tools for Python," (2001-2018). [Online; accessed 2018-04-06].

[27] Gorbach, A. V. and Skryabin, D. V., "Light trapping in gravity-like potentials and expansion of supercontinuum spectra in photonic-crystal fibres," Nature Photonics 1, 653 (2007).

[28] Akhmediev, N. and Karlsson, M., "Cherenkov radiation emitted by solitons in optical fibers," Phys. Rev. A 51, 2602-2607 (1995).

[29] Demircan, A., Amiranashvili, S., Brée, C., Mahnke, C., Mitschke, F., and Steinmeyer, G., "Rogue wave formation by accelerated solitions at an optical event horizon," Appl. Phys. B 115, 343-354 (2014).

[30] Linden, S., Kuhl, J., and Giessen, H., [Frequency-Resolved Optical Gating: The Measurement of Ultrashort Laser Pulses], ch. XFROG - Cross-correlation Frequency-resolved Optical Gating, 313-322, Springer US, Boston, MA (2000). 